\title{
Pharyngeal electrical stimulation device for the treatment of neurogenic dysphagia: technology update
}

This article was published in the following Dove Press journal:

Medical Devices: Evidence and Research

\section{Domenico A Restivo' \\ Shaheen Hamdy²}

'Neurological Unit, Garibaldi Hospital, Catania, Italy; ${ }^{2} \mathrm{School}$ of Translational Medicine-Inflammation Sciences, Faculty of Medical and Human

Sciences, University of Manchester Salford Royal Hospital, Salford, UK
Correspondence: Domenico A Restivo Neurological Unit, Garibaldi Hospital, Piazza S. Maria di Gesù 5, 95I00 Catania, Italy

Tel +39095 7593909

Fax +39095 7593934

Email darestivo@libero.it

\begin{abstract}
Neurogenic dysphagia (ND) can occur in patients with nervous system diseases of varying etiologies. Moreover, recovery from ND is not guaranteed. The therapeutic approaches for oropharyngeal ND have drastically changed over the last decade, mainly due to a better knowledge of the neurophysiology of swallowing along with the progress of neuroimaging and neurophysiological studies. For this reason, it is a priority to develop a treatment that is repeatable, safe, and can be carried out at the bedside as well as for outpatients. Pharyngeal electrical stimulation (PES) is a novel rehabilitation treatment for ND. PES is carried out via location-specific intraluminal catheters that are introduced transnasally and enable clinicians to stimulate the pharynx directly. This technique has demonstrated increasingly promising evidence in improving swallowing performance in patients with ND associated with stroke and multiple sclerosis, probably by increasing the corticobulbar excitability and inducing cortical reorganization of swallowing motor cortex. In this article, we update the reader as to both the physiologic background and past and current studies of PES in an effort to highlight the clinical progress of this important technique.
\end{abstract}

Keywords: pharyngeal electrical stimulation, swallowing, neurogenic dysphagia, swallowing motor cortex, pharynx, catheter

\section{Introduction}

Swallowing is defined as the semiautomatic motor action of the muscles of respiratory, oropharyngeal, and gastrointestinal tracts that propels the food from the oral cavity to the stomach and protects the airway from the unwanted entry of food, liquids, and other substances. ${ }^{1}$ During a swallow, different levels of the central nervous system from the cerebral cortex to the medulla oblongata are involved. For this reason, swallowing is one of the most important bodily functions. Dysphagia is defined as an impairment of this complex and integrated sensorimotor system. Neurogenic dysphagia (ND) typically occurs in patients with neurological diseases of varying etiologies, and it is associated with high mortality, morbidity, and social costs because of the increased risk of aspiration pneumonia and its sequelae. ${ }^{2-7}$

Recovery of ND in most of the central nervous system disorders is unpredictable and unlikely in some of the more progressive disorders. Moreover, even if it is probable that interventions that alter the overall course of the disease, such as the disease modifiers in multiple sclerosis (MS) and in Parkinson's disease and the reperfusion agents in stroke that may prevent or delay dysphagia, at this moment no pharmacological treatment has been shown to be directly effective at treating ND. In the past, several 
rehabilitation therapies have been proposed for ND. However, two decades of research have fueled cautious optimism for rehabilitative strategies for dysphagia therapy. Despite this optimism, optimal protocols have yet to be established. ${ }^{8} \mathrm{Cur}$ rently, the clinical guidelines for the management of ND are mainly represented by compensatory strategies or postural changes in order to reduce the risk of complications. ${ }^{9}$

The therapeutic approach for oropharyngeal ND has drastically changed over the last decade or so, mainly due to a better knowledge of the neurophysiology of swallowing along with the progress made from neuroimaging and neurophysiological studies. Moreover, better insight into the possible physiological mechanisms by which interventional therapies may work has been provided by studies of peripheral electrical stimulation in humans.

Pharyngeal electrical stimulation (PES) is a new therapeutic neurostimulation tool for treating ND that has led to a recent growing clinical interest.

In the present review, after a brief discussion on the neurophysiology of the central control of swallowing, we aim to point out the current literature on the use of PES, for oropharyngeal dysphagia, and to describe the physiological basis, the technical methodology, and the clinical application of this new neurostimulation technique.

\section{Neurophysiology of swallowing}

Swallowing has been described as a complex process regulated by a trigger center in the brainstem, the "central pattern generator" (CPG), which receives inputs from mucosal receptors and from muscles as well as from the cerebral cortex, and thus coordinates the swallowing process. ${ }^{1}$ The CPG for deglutition is located in the medulla oblongata, corresponding to the area of the nucleus tractus solitarius (NTS) (Figure 1). ${ }^{16}$ The CPG consists of four units, two per side, which receive both ascending and descending inputs, and lead the final stage of the swallow. The NTS receives afferent fibers from the nucleus ambigus, which in turn sends efferent fibers to the nuclei of the Vth, VIIth, IXth, Xth, and XIIth cranial nerves, that control the most important muscles involved in swallowing. ${ }^{1}$ Moreover, NTS receives sensitive inputs from oral, pharyngeal, and laryngeal mucosa as well as from upper cerebral areas and can modulate swallowing dependent on bolus properties such as size, texture, and temperature. A lesion interrupting these connections can therefore cause dysphagia. Furthermore, the two hemi-CPGs are tightly synchronized and output the coordinate contraction of the bilateral muscles of the oropharyngeal region. Anatomical connections mediated by fibers crossing the midline have been found to exist between the two CPGs. ${ }^{1}$

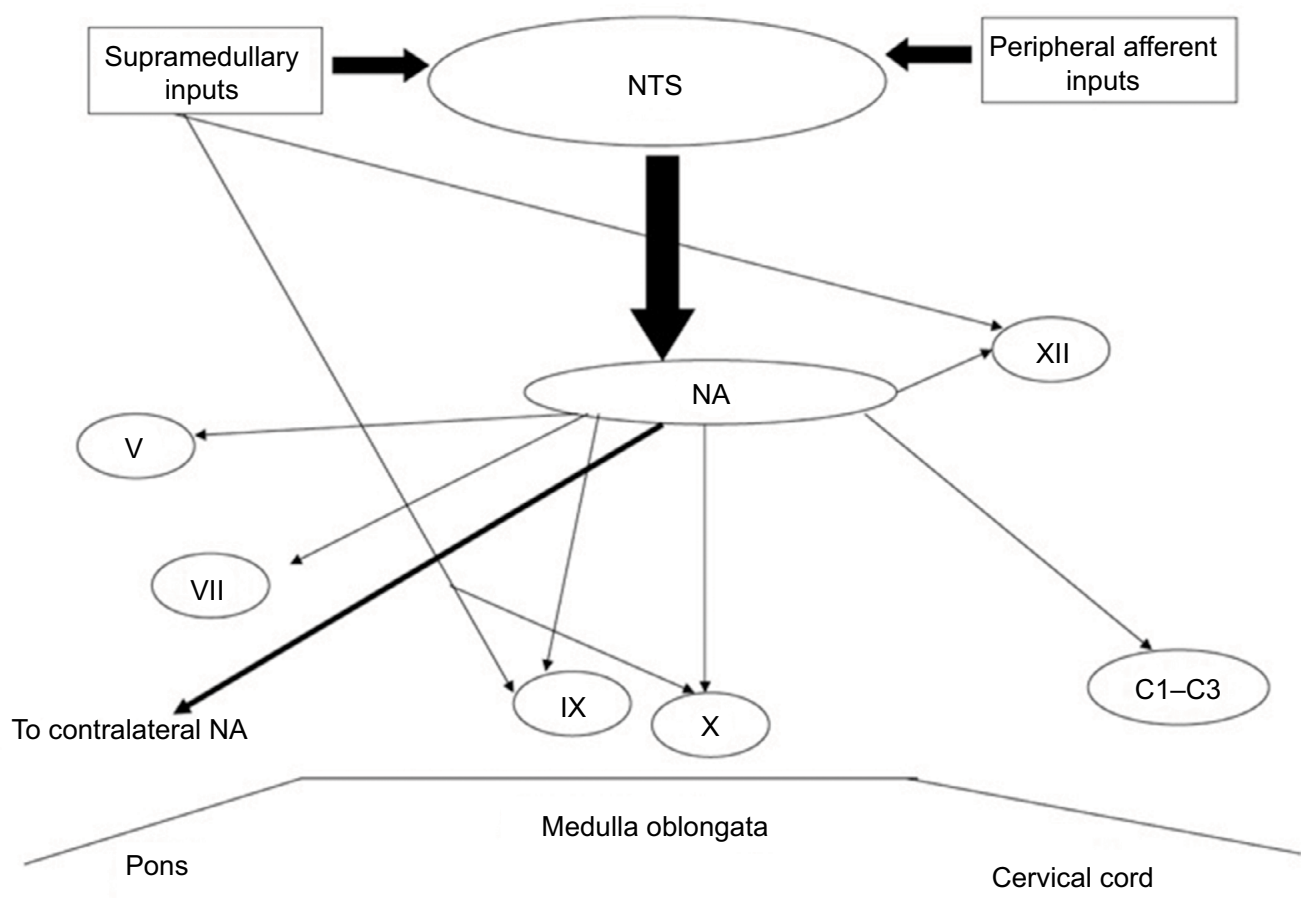

Figure I The CPG for deglutition that is located in the medulla oblongata, corresponding to the area of the NTS and its connections with the NA and the nuclei of the cranial nerves (Vth, VIlth, IXth, Xth, and XIIth) and the upper cervical spine (CI-C3) involved in swallowing.

Abbreviations: CPG, central pattern generator; NA, nucleus ambigus; NTS, nucleus tractus solitarius. 
In recent years, the role of cerebral cortex in swallowing control has attracted increased interest and has been the subject of much research. ${ }^{10}$ In studies on anesthetized animals, artificial cortical stimulation has been shown to induce complete swallow responses. These results provided evidence of a bilateral cortical control of swallowing musculature. ${ }^{11-13}$ Using transcranial magnetic stimulation (TMS), Hamdy and colleagues mapped the musculature involved in swallowing control, mylohyoid, pharyngeal, and esophageal muscles in healthy subjects. ${ }^{14,15}$ These results demonstrated that human swallowing musculature is somatotopically represented bilaterally within the motor and premotor cortex but displays an interhemispheric asymmetry. This asymmetry is independent of handedness, indicating the presence of a hemispheric dominance for swallowing in the cerebral cortex. ${ }^{14,15}$ The corticobulbar connections originate in these areas and project to the ipsilateral and contralateral brainstem nuclei of the main cranial nerves involved in swallowing. ${ }^{14,15}$

In recent years, neuroimaging and neurophysiological studies have shed further light on this process and showed that the most consistent areas that are activated in the forebrain are the primary sensorimotor cortex, sensorimotor integration areas, part of the premotor cortex, the anterior insula cortex, the frontoparietal operculum, the cingulate cortex, and the supplementary motor areas. ${ }^{16}$ Moreover, it has been demonstrated that there is a high probability of bidirectional connections between the supplementary motor area and the primary sensorimotor cortex during swallowing. ${ }^{17}$ Specifically, TMS studies have demonstrated in dysphagic patients that recovered swallowing function after a focal cerebral lesion, such as unilateral stroke, was associated with an increase of corticopharyngeal excitability and cortical map size in the unaffected hemisphere. Conversely, no change has been observed in patients with persistent dysphagia or in nondysphagic patients. These findings seem to underline the role of mechanisms of cortical reorganization in the unaffected hemisphere that are very likely associated with cerebral plasticity. ${ }^{15,18}$

\section{PES and underlying mechanisms}

Given these mechanistic insights, it has been hypothesized that targeting brain plasticity in the swallowing system would be an important method to induce swallowing recovery. Indeed, it was speculated that the effect of PES in swallowing recovery may be due to an increase or triggering of stimulusmediated cortical rearrangements. ${ }^{18,19}$ In order to answer these questions, the first experiments on intraluminal pharyngeal stimulation were carried out by Hamdy's group. ${ }^{15,18}$ These authors introduced a neurophysiological technique in which electrical stimuli with a specific frequency were applied to the pharyngeal mucosa by using bipolar-ring specifically built intraluminal electrodes. ${ }^{15,18}$

Eight healthy volunteers received stimuli at different frequencies, intensities, and durations through a platinum ring pharyngeal electrode built into an intraluminal catheter inserted transorally or transnasally in order to determine which pattern produced the largest effect on corticopharyngeal excitability. Electromyographic (EMG) responses evoked in the pharynx by TMS were tested before stimulation, and then immediately, 30 minutes afterwards, and $60 \mathrm{~min}$ utes afterwards. Stimulation was uniformly performed for 10 minutes at different frequencies $(1,5,10,20$, or $40 \mathrm{~Hz})$. For each frequency, the intensity was set at $75 \%$ of the maximum tolerated so that the sensation was approximately equal. It was observed that stimulation at 1 or $5 \mathrm{~Hz}$ increased the cortical excitability as determined by an increase in the amplitude of motor evoked potentials (MEPs) response following TMS. Conversely, stimulation of 10, 20, and $40 \mathrm{~Hz}$ decreased excitability. Stimulation at $5 \mathrm{~Hz}$ demonstrated the largest effect that was maximum 30 and 60 minutes after the end of stimulation. It was also demonstrated that the higher the intensity of stimulation, the higher the increase in corticobulbar excitability, with larger responses at 60 minutes compared with immediately after stimulation. ${ }^{15,18}$ Contemporary recordings of MEP from the hand (thenar) muscles, showed no changes in excitability of the hand motor area, indicating a focal effect of PES on corticopharyngeal excitability. These initial experiments suggested that applications of PES (10 minutes, at $5 \mathrm{~Hz}$, at $75 \%$ maximum tolerated intensity) induced the maximal corticobulbar excitability. ${ }^{15,18}$ For these reasons, all the subsequent experiments were carried out with $5 \mathrm{~Hz}$ stimulation. ${ }^{18}$

The protocol was repeated with this pattern of stimulation with additional MEP recordings at 90, 120, and 150 minutes after the stimulation was stopped. Maximal facilitation was observed at 60 and 90 minutes. In a further experiment, it was observed that PES was able to induce changes in the topographic representation of the corticobulbar projections to the pharynx, demonstrated by an increase of the corticopharyngeal map in the scalp. Moreover, functional magnetic resonance imaging studies by the same group, demonstrated that PES can change the pattern of brain activation observed during normal wet swallows. Moreover, more importantly, these paradigms applied to dysphagic hemiplegic stroke patients resulted in a faster initiation of each swallow and a reduction in the frequency of aspiration for at least 1 hour 
after stimulation. ${ }^{18}$ These cortical excitability increases, and the consequent changes in neural connections organization are a feature of "cortical plasticity."19

\section{Possible mechanisms of PES}

The possible mechanisms underlying the effects of PES in increasing corticopharyngeal excitability as well as in improving swallowing performances are still not completely clear. The effects induced by PES on cortical plasticity, from a clinical point of view, seem to indicate that the increase in corticobulbar excitability, and consequently the swallowing recovery is "stimulus-driven." It is very likely that the effect of pharyngeal stimulation is due to the sensory afferents lying in the naso- and oropharyngeal mucosa. ${ }^{20,21}$ The stimulation of these fibers, which represent the sensory afferents of the glossopharyngeal nerve (IXth) and the vagus nerve (Xth, pharyngeal branch), are directly connected to the NTS. These sensory inputs are transmitted to other upper brainstem zones as well as to subcortical and cortical areas. It has been reported that the convergent activity of different pharyngeal afferent signals can increase the swallowing sensorimotor cortex excitability. ${ }^{22,23}$

\section{Technology}

In the first experiments, the stimulation electrodes, after being positioned in the pharynx (Figure 2), were connected to a commercially available constant current electrical stimulator and trigger generator, enabling the examiner to set the stimulation for a large range of frequencies and intensities. With this kind of stimulation, it is possible to deliver a "real" stimulation, or a "sham" stimulation to the pharynx.

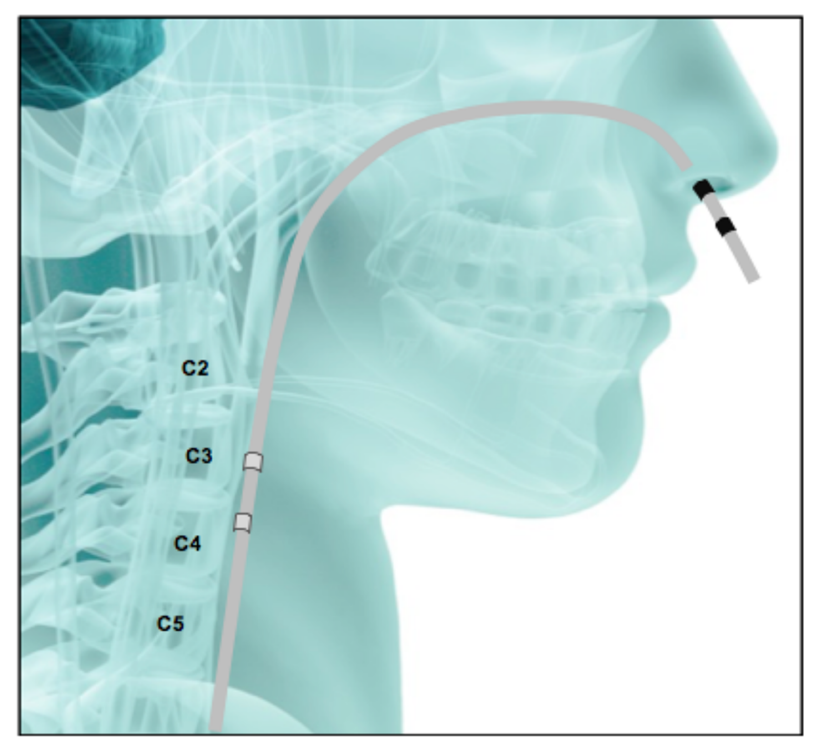

Figure 2 The transit of the electrode for pharyngeal stimulation from the site of introduction to its position in the pharyngeal wall.

Notes: Figure courtesy of Phagenesis ${ }^{\circledR}$, Manchester, UK. C2 refers to nasopharynx; C3 refers to oropharynx; C4 refers to laryngopharynx; C5, refers to esophagus.
For "sham" stimulation, the same catheter as that for "real" stimulation is used, but no current is passed. ${ }^{19}$

After about 20 years of research on PES by the Hamdy group, demonstrating the efficacy and safety of this kind of stimulation in the treatment of dysphagia, a specific commercial device for PES has been developed and is now available. The device is named "Phagenyx ${ }^{\circledR}$ " (Phagenesis Ltd, Manchester, UK), (Figure 3). It consists of a base station and a treatment catheter, which when used in combination enables the surgeon to perform personal treatment for dysphagic patients. The base station delivers the optimal stimulation for patients, and at the same time, possesses a setting limited to a specific range of intensities and frequencies that have been demonstrated to be the most effective and safe in dysphagia treatment. The base station also records and stores patient information to avoid errors in treatment. It is battery-powered, portable, and operated through a touch-screen interface. The stimulation is delivered to the patient's pharynx through the Phagenyx treatment catheter, which is connected with the base station and is introduced through the nose. There are two electrodes on the outer surface of the catheter through which the stimulation is delivered. Guide marks ensure the electrodes are positioned correctly for treatment. The electrodes usually lie $3 \mathrm{~cm}$ above the upper esophageal sphincter. The catheter design incorporates an optional feeding tube that can be used for the administration of nutrition, fluids, and medications.

\section{Clinical application}

PES has been demonstrated to show plausible promise in poststroke dysphagia. ${ }^{18,24-26}$ In a study on poststroke patients, PES treatment each day for 3 days improved airway protection compared with controls and also reduced aspiration and improved feeding status, consequently resulting in a shorter time to hospital discharge. ${ }^{24}$ In another study aimed at evaluating the long-term effect of PES in poststroke dysphagia, PES demonstrated accelerated swallowing recovery over the first 2 weeks poststimulation. ${ }^{25}$ A recent meta-analysis of three randomized controlled trials (RCTs) on PES treatment for poststroke dysphagia concluded that PES is associated with less radiological aspiration and clinical dysphagia, leading to possibly reduced length of stay in hospital. ${ }^{26}$ In a recent RCT, 162 patients with subacute ischemic or hemorrhagic stroke and dysphagia were enrolled. PES or sham stimulation was delivered for 3 consecutive days. The primary outcome was swallowing safety at 2 weeks. Secondary outcomes included dysphagia severity, functional quality of life, and serious adverse events at 6 and 12 weeks. The results of this study showed that PES was safe (no serious adverse device-related events occurred) but did not show significant superiority on 


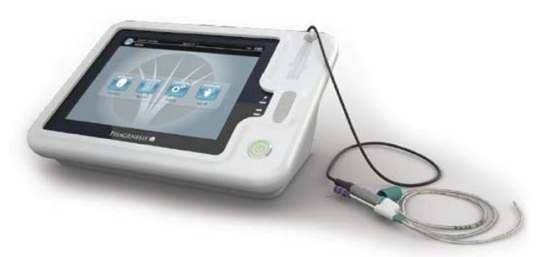

Figure 3 The Phagenyx ${ }^{\circledR}$ system.

Note: Figure courtesy of Phagenesis ${ }^{\circledR}$, Manchester, UK

aspiration scores as compared to sham stimulation. However, several factors including the enrollment of patients with mild dysphagia, potential undertreatment with PES, and possible active stimulation on control patients may have contributed to these neutral results. ${ }^{27}$ In a recent pilot RCT study of 30 tracheotomized dysphagic, more severe stroke patients, PES enhanced remission of dysphagia in $75 \%$ of poststroke patients and consequently enabled decannulation. ${ }^{28}$

Restivo et al, ${ }^{19}$ investigated the PES effect on swallowing recovery in 20 patients affected with MS and severe dysphagia. Twenty dysphagic patients were randomized to receive $5 \mathrm{~Hz}$ "real" PES or "sham" pharyngeal stimulation for 10 minutes. Patents who received "real" PES showed a significant improvement in all the swallowing outcome measures analyzed as compared with those receiving "sham" stimulation. ${ }^{19}$ This preliminary study suggests a potential benefit of PES for MS-associated dysphagia. ${ }^{19}$

Table 1 shows a summary of developmental and investigational trials of the Phagenyx System.

\section{Advantages and limitations}

Overall, PES seems to be a safe and potentially effective rehabilitation tool with no important stimulation-induced adverse events reported. However, even if this neurophysiological technique has been demonstrated to be very safe, the introduction of the catheter transnasally may be considered unpleasant for some patients. However, most dysphagic patients will require nasogastric feeding, so it is in the patient's best interest for the patients to utilize such a technique should it show both efficacy and effectiveness in future trials. The major limitation of PES treatment is the exact localization of the site of stimulation in the pharynx. In fact, given the conformation of the pharyngeal wall, it is not possible to know the side of the catheter position in the pharynx, and consequently the side of stimulation. However, it remains unclear as to whether knowledge of the side of stimulation has any clinical relevance to treatment effect.

\section{Conclusion}

Treatment options for dysphagia associated with neurological disorders are currently limited or absent. No pharmacological treatment has been shown to be effective in improving swallowing performances, and, furthermore, while many rehabilitative treatments have been developed in recent years, the most effective protocol has yet to be demonstrated. In fact, stimulus delivery is difficult to standardize and is therefore applied in a variable manner that may dilute any beneficial effect. A more objective definition and standardization of the stimulation parameters is thus required to evaluate any possible benefits from these techniques and determine the cost-benefit ratio. For this reason, it is a priority to develop a treatment that is repeatable, safe, and can be carried out at the bedside as well as in the outpatient setting.

In comparison with other techniques, PES has been demonstrated to be a promising treatment option for dysphagia in stroke and MS patients. The treatment is repeatable and quite easy to perform at the bedside. Although in a recent large trial of dysphagic stroke patients, PES was unable to

Table I Summary of developmental and investigational trials of the PES

\begin{tabular}{|c|c|c|c|}
\hline Study type & Purpose & Subjects & $\mathbf{N}$ \\
\hline Developmental & Establish optimal parameters for PES & Healthy volunteers & 8 \\
\hline Feasibility RCT & Assess short-term effects of PES in altering swallowing after stroke, acute period & Stroke pts with dysphagia & 16 \\
\hline Experimental & Assess use of PES to reverse virtual lesions & Healthy volunteers & 13 \\
\hline Dose response & $\begin{array}{l}\text { Establish the optimal number of treatment sessions based on safety and effectiveness in } \\
\text { acute stroke }\end{array}$ & Stroke pts with dysphagia & 22 \\
\hline Phase Ila RCT & $\begin{array}{l}\text { Assess safety and effectiveness of optimized PES treatment regimen in acute stroke; } \\
\text { 2-week follow-up }\end{array}$ & Stroke pts with dysphagia & 28 \\
\hline Phase Ilb RCT & $\begin{array}{l}\text { Assess safety and effectiveness of optimized PES treatment regimen in acute stroke; } \\
\text { 3-month follow-up }\end{array}$ & Stroke pts with dysphagia & 36 \\
\hline Phase III RCT & Assess PES in acute stroke in a study powered for significance & Stroke pts with dysphagia & 133 \\
\hline Phase II RCT & Assess use of PES to facilitate early tracheostomy decannulation & Stroke pts with dysphagia & 30 \\
\hline Exploratory & Assess utility of PES in chronic, persistent dysphagia & Stroke pts with dysphagia & 6 \\
\hline Feasibility RCT & Assess safety and effectiveness of PES in MS & MS pts with dysphagia & 20 \\
\hline
\end{tabular}

Abbreviations: MS, multiple sclerosis; PES, pharyngeal electrical stimulation; pts, patients; RCT, randomized controlled trial. 
demonstrate superiority on radiological aspiration or on clinical dysphagia, several factors including undertreatment of patients receiving PES may have contributed to these results. Indeed, the above results differ from the findings of a previous meta-analysis of three smaller RCTs, which showed an effect at 2 weeks compared to sham. Further studies selecting stroke patients with more severe dysphagia or those requiring intensive care ventilation need to be designed. In addition, studies evaluating the effect of PES on dysphagia due to different neurological disorders, as well as the association of PES in combination with other rehabilitative treatments, are necessary.

\section{Disclosure}

S Hamdy is a director, chief scientific officer, and shareholder in the company Phagenesis Ltd, which is involved in the medical device sector for dysphagia and markets Phagenyx ${ }^{\mathrm{TM}}$. The authors report no other conflicts of interest in this work.

\section{References}

1. Jean A. Brain steam control of swallowing: neuronal network and cellular mechanisms. Physiol Rev. 2001;81:929-969.

2. Abraham S, Scheinberg A, Smith CR, La Rocca NG. Neurologic impairment and ability status in outpatients with multipla sclerosis reporting dysphagia symptomatology. J Neurol Rehab. 1997;11:7-13.

3. Bergamaschi R, Crivelli P, Rezzani C, et al. The DYMUS questionnaire for the assessment of dysphagia in multiple sclerosis. J Neurol Sci. 2008;5;269(1-2):49-53.

4. Restivo DA, Marchese-Ragona R, Patti F, et al. Botulinum toxin improves dysphagia associated with multiple sclerosis. Eur J Neurol. 2011;18(3):486-490.

5. Restivo DA, Palmeri A, Marchese-Ragona R. Botulinum toxin for cricopharyngeal dysfunction in Parkinson's disease. $N$ Engl J Med. 2002;346:1174-1175.

6. Alfonsi E, Versino M, Merlo IM, et al. Electrophysiological pattern of oro-pharyngeal swallowing in parkinsonian syndromes. Neurology. 2007;68(8):583-589.

7. Restivo DA, Marchese-Ragona R, Staffieri A, De Grandis D. Successful botulinum toxin treatment of dysphagia in oculopharyngeal dystrophy. Gastroenterology. 2000;119:1416.

8. Easterling C. 25 Years of dysphagia rehabilitation: what have we done, what are we doing, and what are we going? Dysphagia. 2017;32(1):50-54.

9. Speyer R, Baijens L, Heijnen M, et al. Effects of therapy in oropharyngeal dysphagia by speech and language therapists: a systematic review. Dysphagia. 2010;25(1):40-65.

10. Michou E, Hamdy S. Cortical input in control of swallowing. Curr Opin Otolaryngol Head Neck Surg. 2009;17(3):166-171.
11. Sumi T. Some properties of cortically-evoked swallowing and chewing in rabbits. Brain Res. 1969;15(1):107-120.

12. Martin RE, Kemppainnen P, Masuda Y, et al. Features of cortically evoked swallowing in the awake primate (Macaca fascicularis). J Neurophysiol. 1999;82(3):1529-1541.

13. Hamdy S, Xue S, Valdez D, et al. Induction of cortical swallowing activity by transcranial magnetic stimulation in anaesthetized cat. Neurogastroenterol Motil. 2001;13(1):65-72.

14. Hamdy S, Aziz Q, Rothwell JC, et al. Recovery of swallowing after dysphagic stroke relates to functional reorganization in the intact motor cortex. Gastroenterology. 1998;115:1104-1112.

15. Hamdy S, Rothwell JC, Aziz Q, Singh KD, Thompson DG. Long-term reorganization of human motor cortex driven by short-term sensory stimulation. Nat Neurosci. 1998;1:64-68.

16. Soros P, Inamoto Y, Martin RE. Functional brain imaging of swallowing: an activation likelihood estimation meta-analysis. Hum Brain Mapp. 2009;30(8):2426-2439.

17. Mihai PG, Otto M, Platz T, et al. Sequential evolution of cortical activity and effective connectivity of swallowing using fMRI. Hum Brain Mapp. 2014;35(12):5962-5973.

18. Fraser C, Power M, Hamdy S, et al. Driving plasticity in human adult motor cortex is associated with improved motor function after brain injury. Neuron. 2002;34:831-840.

19. Restivo DA, Casabona A, Centonze D, Marchese-Ragona R, Maimone D, Pavone A. Pharyngeal electrical stimulation for dysphagia associated with multiple sclerosis: a pilot study. Brain Stim. 2013;6(3): 418-423.

20. Steele CM, Miller AJ. Sensory input pathways and mechanisms in swallowing: a review. Dysphagia. 2010;25:323-333.

21. Sweazey RD, Bradley R. Response of lamb nucleus of the solitary tract neurons to chemical stimulation of the epiglottis. Brain Res. 1988;439:195-210.

22. Sweazey RD. Distribution of aspartate and glutamate in the nucleus of the solitary tract in the lamb. Exp Brain Res. 1995;105:241-253.

23. Gow D, Rothwell J, Hobson A, Thompson D, Hamdy S. Induction of long-term plasticity in human swallowing cortex following repetitive cortical stimulation. Clin Neurophysiol. 2004;115:1044-1051.

24. Jayasekeran V, Singh S, Tyrrell P, et al. Adjunctive functional pharyngeal electrical stimulation reverses swallowing disability after brain lesions. Gastroenterology. 2010;138(5):1737-1746.

25. Vasant DH, Michou E, O'Leary N, Vall A, Mistry S, Hamdy S; Greater Mancher Stroke Research Network. Pharyngeal Electrical Stimulation in dysphagia poststroke: A prospective, randomized, single-blinded interventional study. Neurorehabil Neural Repair. 2016;30(9): 366-375.

26. Scutt P, Lee HS, Hamdy S, Bath PM. Pharyngeal Electrical Stimulation for treatment of poststroke dysphagia: individual patient data Metaanalysis of Randomised Controlled Trials. Stroke Res Treat. 2015; 429052.

27. Bath PM, Scutt P, Love J, et al; Swallowing Treatment Using Pharyngeal Electrical Stimulation (STEPS) Trial Investigators. Pharyngeal electrical stimulation for treatment of dysphagia in subacute stroke: A Randomized Controlled Trial. Stroke. 2016;47:1562-1570.

28. Suntrup S, Marian T, Schröder JB, et al. Electrical pharyngeal stimulation for dysphagia treatment in tracheostomized stroke patients: a randomized controlled trial. Intensive Care Med. 2015;41(9):1629-1637.
Medical Devices: Evidence and Research

\section{Publish your work in this journal}

Medical Devices: Evidence and Research is an international, peerreviewed, open access journal that focuses on the evidence, technology, research, and expert opinion supporting the use and application of medical devices in the diagnosis, monitoring, treatment and management of clinical conditions and physiological processes. The identification of novel

\section{Dovepress}

devices and optimal use of existing devices which will lead to improved clinical outcomes and more effective patient management and safety is a key feature. The manuscript management system is completely online and includes a quick and fair peer-review system. Visit http://www. dovepress.com/testimonials.php to read real quotes from authors. 